\title{
Parotid Gland Solitary Fibrous Tumor Presenting as a Long Duration Mass: A Case Report
}

\author{
Khadijah Abdulhaleem $\mathbb{D}^{1},{ }^{1}$ Mohammad Anas Dababo $\mathbb{D}^{2},{ }^{2}$ and Eyas Othman $\mathbb{D}^{3}$ \\ ${ }^{1}$ Department of Central Military Laboratory and Blood Bank, Prince Sultan Military Medical City, Riyadh, Saudi Arabia \\ ${ }^{2}$ Department of Pathology and Laboratory Medicine, King Faisal Specialist Hospital and Research Centre, Riyadh, Saudi Arabia \\ ${ }^{3}$ Department of Otolaryngology, King Faisal Specialist Hospital and Research Centre, Riyadh, Saudi Arabia
}

Correspondence should be addressed to Khadijah Abdulhaleem; kh.abdulhaleem@gmail.com

Received 12 November 2021; Accepted 28 January 2022; Published 24 February 2022

Academic Editor: Evelina Miele

Copyright (c) 2022 Khadijah Abdulhaleem et al. This is an open access article distributed under the Creative Commons Attribution License, which permits unrestricted use, distribution, and reproduction in any medium, provided the original work is properly cited.

\begin{abstract}
The solitary fibrous tumor (SFT) is a tumor of uncertain histogenesis, affecting deep soft tissues, particularly the pleura (pulmonary) and extrapulmonary sites including thighs, retroperitoneum, other serosal surfaces, and cranial and spinal meninges. SFT and hemangiopericytoma are now considered the same entity, with general agreement on referring to this group of tumors as "SFT." SFTs are generally benign tumors with small subsets of malignant ones. Moreover, they are well-circumscribed with a good prognosis after surgical resection. SFTs are uncommon in the head and neck and are quite rare in the parotid gland region. Here, we present a case of a 48-year-old female with SFT of the parotid gland region; the diagnosis was confirmed by positive immunohistochemical staining for Bcl-2, CD34, and STAT6. STAT6 immunohistochemistry is sensitive and specific for SFTs.
\end{abstract}

\section{Introduction}

A solitary fibrous tumor (SFT) is a rare soft tissue spindle cell neoplasm primarily affecting the pleura. In 1931, Klemperer and Rabin first described the tumor as a primary neoplasm involving the pleura and classified plural tumors into diffused and localized types [1]. Lietaud, in 1767, and Wagner, in 1870, were the first to report SFT in the pleura and noted the localized nature of the lesion [2]. In recent years, SFT has been reported in a variety of extrapleural sites. SFT in the head and neck region accounts for approximately 10-15\% of all SFT cases [3]. The most common sites affected are the nasal, paranasal, nasopharynx, larynx, and oral cavities and the orbit [4]. Other head and neck sites include major salivary glands, especially the parotid gland, where 35 cases have been reported in the English literature. Parotid SFT was first described in1995 by Hanau and Miettinen [5]. Since SFTs of the parotid gland are unusual, these tumors may be misdiagnosed as other more common tumors in salivary glands. Herein, we report an additional case of SFT of the parotid gland without recurrence in a twelve-month follow-up.

\section{Case Presentation}

A 48-year-old female presented with a painless swelling in the right parotid gland region for 12 years. The mass was slowly increasing in size, especially over the past several months. Moreover, there was no history of constitutional symptoms. Her past medical/surgical history was unremarkable. Physical examination showed a right-sided parotid region, nontender, firm, immobile, smoothly countered mass without overlying erythema. There was no facial nerve paralysis or paresthesia. Cervical lymph nodes were not palpable.

Magnetic resonance imaging (MRI) for the head and neck showed a large mildly lobulated lesion centered within the superficial lobe of the right parotid gland with extension anterior to the right mandibular ramus into the masticator space (Figure 1). The mass in the parotid gland measured approximately $4.3 \times 3.2 \times 5.2 \mathrm{~cm}$ in the anteroposterior, transverse, and craniocaudal dimensions, respectively. The 


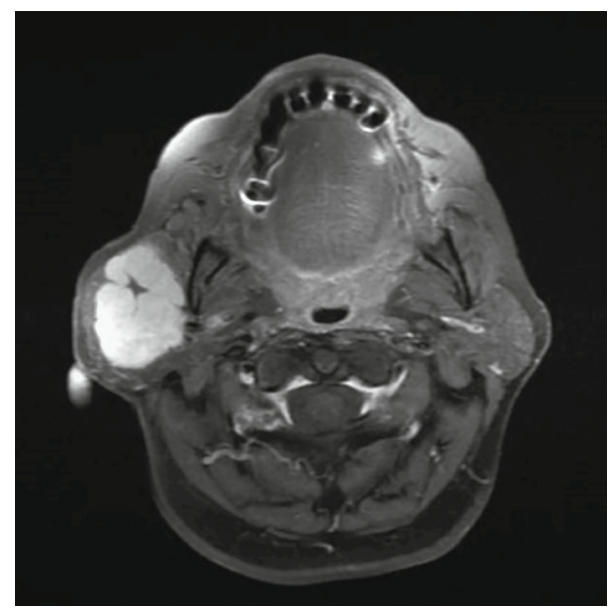

Figure 1: An axial MRI image demonstrating a lobulated lesion centered within the superficial lobe parotid gland (right side).

tumor has a T2 hyperintense signal and a T1 intermediate signal with avid enhancement. The tumor is relatively wellmarginated with preservation of the surrounding parotid parenchyma. There is no focal lesion in the left parotid gland or the submandibular glands.

Fine-needle aspiration (FNA) was performed, which yielded mainly blood aspirate and no diagnostic material. The patient underwent a right total parotidectomy without complications.

Macroscopically, the parotid gland measures $7.0 \times 5.0 \times$ $3.0 \mathrm{~cm}$. Serial sections revealed a multilobulated nodular, pale to tan, rubbery mass measuring $6.5 \times 5.0 \times 2.3 \mathrm{~cm}$, identified on the inked margin. Microscopically, the tumor was relatively well-defined and showed a thin fibrous capsule (Figure 2). There was unremarkable salivary gland parenchyma in the background, with the tumor present at the inked margin of the sample. The tumor showed a normal salivary duct at the edge of the lesion. The neoplasm was composed of a lobulated proliferation of spindleshaped cells without a defined architectural pattern forming short fascicles with interspersed thin collagen bundles (Figure 3). The spindle cells were monotonous, rounded to oval nuclei with coarse nuclear chromatin distribution and tapering eosinophilic cytoplasm. Delicate thin-walled vascular spaces, with hemangiopericytoma-like vasculature, were observed at the periphery (Figure 4). A few lymphocytes and scattered mast cells were present among the neoplastic cells. Mitosis, necrosis, cellular pleomorphism, or other signs of malignancy were absent.

Immunohistochemical studies were conducted using cytokeratin AE1/AE, EMA, S100, CD34, CD31, CD21, CD23, SMA, Bcl2, CD99, and STAT6. The tumor cells were strongly positive for Bcl2 and CD34 (Figure 5) and showed focal membrane positivity for CD99 and diffuse nuclear positivity for STAT6 (Figure 6), whereas they were negative for cytokeratin AE1/AE3, EMA, S-100 protein, CD21, CD23, SMA, and CD31. These results confirmed our diagnosis of SFT and ruled out other differential diagnoses such as spindle cell carcinoma, spindle cell myoepithelioma, schwannoma, and monophasic synovial sarcoma.

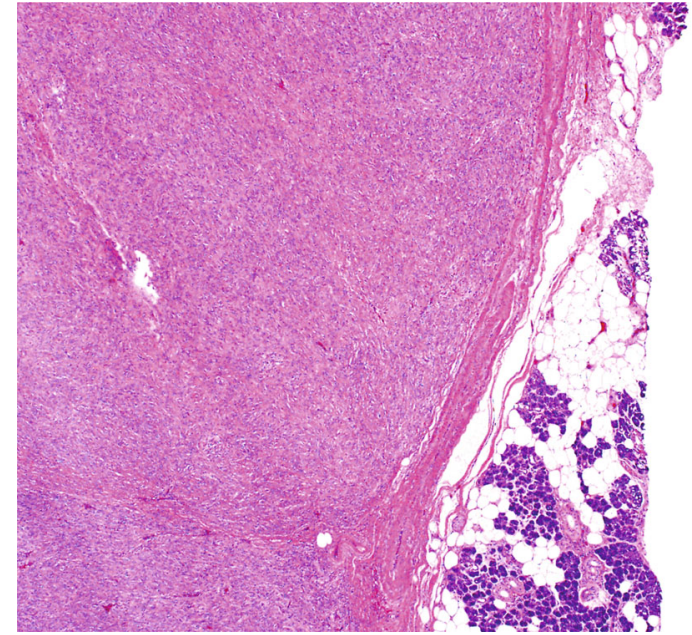

FIgure 2: The lesion with well-defined border and showed a thin fibrous capsule. Unremarkable salivary gland tissue noted in the periphery $(\mathrm{H} \& \mathrm{E}, \times 10)$.

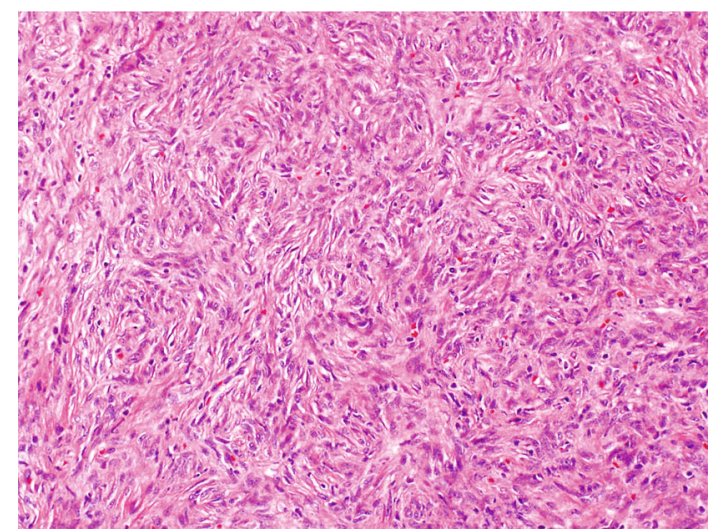

Figure 3: The tumor with spindle-shaped cells without a defined architectural pattern forming short fascicles with interspersed thin collagen bundles $(\mathrm{H} \& \mathrm{E}, \times 20)$.

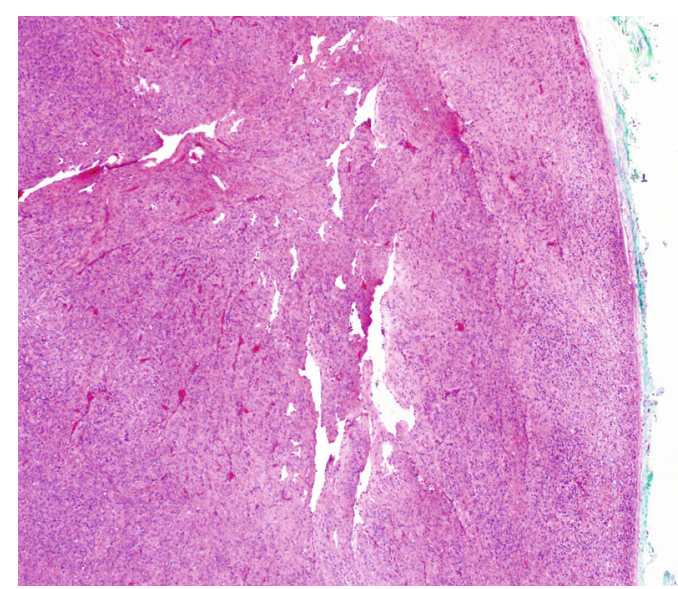

Figure 4: Delicate thin-walled vascular spaces, with hemangiopericytoma-like vasculature, were observed at the periphery $(\mathrm{H} \& \mathrm{E}, \times 10)$. 


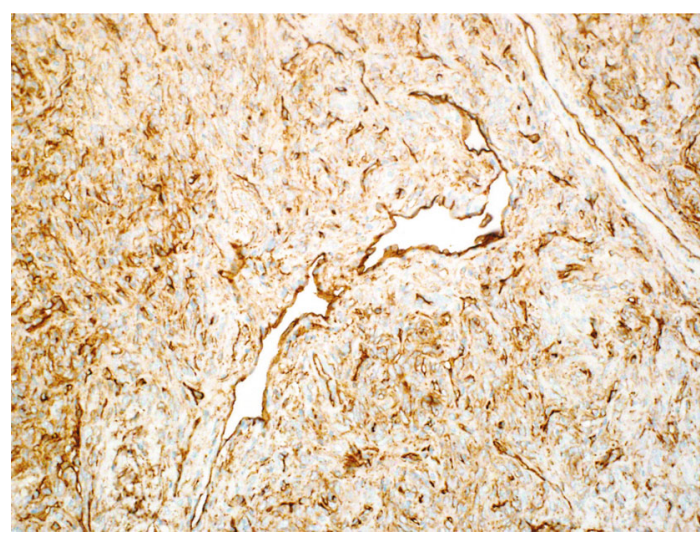

FIgure 5: A diffuse positive immunoreactivity against CD34 $(\times 20)$.

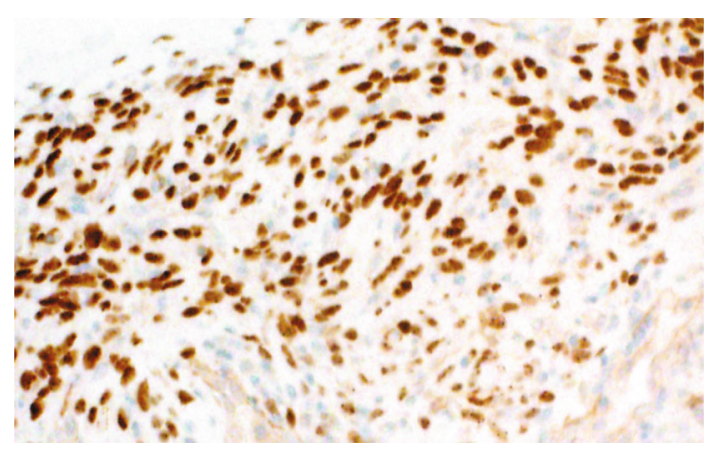

FIGURE 6: Positive immunoreactivity against STAT6, nuclear type positivity $(\times 20)$.

The patient has been followed up for twelve months, with no signs of recurrence or complications.

\section{Discussion}

SFT is a rare neoplasm that involves the intrathoracic and extrathoracic sites. SFTs are derived from pluripotent fibroblastic cells [6]. These tumors of uncertain behavior, locally aggressive, rarely metastasizing, can be malignant under the 2020 WHO classification. SFTs most commonly arise from the intrathorax site, especially affecting the pleura, which account for less than $5 \%$ of all neoplastic diseases [6]. Extrathorax SFTs involve different sites, such as soft tissue, peritoneum, pelvis, genitourinary, and head and neck regions. The salivary gland, especially the parotid gland, is one of the rarely involved regions. Only thirty-five cases of SFT in the parotid gland have been reported in the English literature [2, 4, 5, 7-34]. In 2012, Bauer et al. reviewed the literature for the first 22 cases of SFT in the parotid gland, three of which were malignant [2]. From 2012 to 2016, seven more additional cases were published [25-30]. In 2019, Lim et al. reviewed all reported cases of SFT in the major salivary glands, including the parotid area, from 2004 to 2018 [33]. Thus, including our case, only 36 cases have been reported to date. The pathologists must consider SFT in the differen- tial diagnosis of spindle cell-rich lesions affecting the parotid gland such as spindle cell myoepithelioma, schwannoma, spindle cell carcinoma, low-grade myofibroblastic sarcoma, and monophasic synovial sarcoma. However, when suspected based on morphology, the diagnosis can be relatively straightforward, especially with using immunohistochemical stains. Parotid SFT is most often present as a well-defined, palpable, painless, and slow-growing mass, with a median size of $4.4 \mathrm{~cm}[26,28]$. In their review, Sousa et al. [26] have indicated a slight male predominance $(1.2: 1 \mathrm{M}: \mathrm{F}$ ratio) and a mean age of 49.8 years. SFT affects patients of all age groups ranging from 11 to 79 years and is more common in the left parotid gland [26]. Microscopically, the tumor is composed of spindle to ovoid cells arranged in a short fascicular pattern. The cells have a moderate amount of eosinophilic cytoplasm, centrally located nuclei with open, vesicular nuclear chromatin, and indistinct nucleoli. The stroma shows medium-sized hemangiopericytoma-like vessels, in addition to a collagen matrix, myxoid changes, and some inflammatory cells such as mast cells [26]. Immunohistochemical stains are important to confirm the diagnosis. SFT is positive for vimentin, CD34, Bcl2, and CD99 [26]. CD34 is important and a sensitive marker for SFT, although it may not be positive in all cases [2].

Furthermore, diffuse nuclear expression of STAT6 has been found to be a highly sensitive and specific immunohistochemical marker for SFT [31]. Recently, several studies have detected a recurrent intrachromosomal fusion between the NAB2 and STAT6 genes on chromosome 12 in SFT [31]. These gene fusions became pathognomonic for SFT. To our knowledge, this is the third case report that confirms the diagnosis of parotid SFT by diffuse nuclear positivity of STAT6 by immunohistochemical study. Complete local surgical excision with negative margins is the most important prognostic factor in SFT, and a long-term follow-up is recommended [27]. Similar to our case, Ganly et al. [32] have suggested that the patients with positive surgical margins or whose tumors have a malignant component benefit from postoperative radiotherapy. However, few reports have documented this issue and their follow-up periods were short. In our case, there was a positive microscopic surgical margin. We presented the case to the Head and Neck Oncology Committee and decided that no adjuvant therapy was necessary. Bauer et al. [2] have suggested that the cases of parotid gland SFT with positive margins showed no recurrence to date and follow-up was not required. In our case of parotid SFT, patient age, sex, duration of symptoms, size, and immunohistological findings were consistent with previously reported ranges.

In conclusion, SFT of the parotid gland is a rare neoplasm that occurs most often in middle-aged patients, without a gender predilection, who have symptoms of a mass lesion for a long duration. SFT is diagnosed based on classical histologic and positivity for CD34, Bcl-2, and STAT6 (nuclear pattern), which aid in its distinction from other tumors in the differential diagnosis. A short follow-up period suggests a good prognosis, whether the tumors are histologically benign or malignant when managed by complete surgical excision. 


\section{Data Availability}

The pictures used to support the findings of this study are included within the article and separated in figure files.

\section{Conflicts of Interest}

The authors declare no conflict of interest.

\section{Authors' Contributions}

All authors have read and agreed to the published version of the manuscript.

\section{References}

[1] P. Klemperer and C. B. Rabin, "Primary neoplasms of the pleura," Archives of Pathology, vol. 11, pp. 385-412, 1931.

[2] J. L. Bauer, A. Z. Miklos, and L. D. R. Thompson, "Parotid gland solitary fibrous tumor: a case report and clinicopathologic review of 22 cases from the literature," Head and Neck Pathology, vol. 6, no. 1, pp. 21-31, 2012.

[3] J. S. Gold, C. R. Antonescu, C. Hajdu et al., "Clinicopathologic correlates of solitary fibrous tumors," Cancer, vol. 94, no. 4, pp. 1057-1068, 2002.

[4] M. Kumagai, H. Suzuki, E. Takahashi et al., "A case of solitary fibrous tumor of the parotid gland: review of the literatures," The Tohoku Journal of Experimental Medicine, vol. 198, no. 1, pp. 41-46, 2002.

[5] C. A. Hanau and M. Miettinen, "Solitary fibrous tumor: histological and immunohistochemical spectrum of benign and malignant variants presenting at different sites," Human Pathology, vol. 26, no. 4, pp. 440-449, 1995.

[6] J. F. Chick, N. R. Chauhan, and R. Madan, "Solitary fibrous tumors of the thorax: nomenclature, epidemiology, radiologic and pathologic findings, differential diagnoses, and management," AJR. American Journal of Roentgenology, vol. 200, no. 3, pp. W238-W248, 2013.

[7] J. A. Ferreiro and A. G. Nascimento, "Solitary fibrous tumour of the major salivary glands," Histopathology, vol. 28, no. 3, pp. 261-264, 1996.

[8] J. Sato, K. Asakura, Y. Yokoyama, and M. Satoh, "Solitary fibrous tumor of the parotid gland extending to the parapharyngeal space," European Archives of Oto-Rhino-Laryngology, vol. 255 , no. 1 , pp. 18-21, 1998.

[9] R. B. Brunnemann, J. Y. Ro, N. G. Ordonez, J. Mooney, A. K. El-Naggar, and A. G. Ayala, "Extrapleural solitary fibrous tumor: a clinicopathologic study of 24 cases," Modern Pathology, vol. 12, no. 11, pp. 1034-1042, 1999.

[10] K. Mohammed, G. Harbourne, M. Walsh, and D. Royston, "Solitary fibrous tumour of the parotid gland," The Journal of Laryngology and Otology, vol. 115, no. 10, pp. 831-832, 2001.

[11] M. F. Guerra, C. G. Amat, F. R. Campo, and J. S. Perez, "Solitary fibrous tumor of the parotid gland: a case report," Oral and Maxillofacial Pathology, vol. 94, no. 1, pp. 78-82, 2002.

[12] M. Thompson, L. H. Cheng, J. Stewart, A. Marker, and D. M. Adlam, "A paediatric case of a solitary fibrous tumour of the parotid gland," International Journal of Pediatric Otorhinolaryngology, vol. 68, no. 4, pp. 481-487, 2004.

[13] R. Gerhard, E. R. Fregnani, R. Falzoni, S. A. Siqueira, and P. A. Vargas, "Cytologic features of solitary fibrous tumor of the parotid gland. A case report," Acta Cytologica, vol. 48, no. 3, pp. 402-406, 2004.

[14] M. L. Suarez Roa, L. M. R. G. Rivera, G. A. Meneses, M. Granados-Garcia, and T. A. Mosqueda, "Solitary fibrous tumor of the parotid region. Report of a case and review of the literature," Medicina Oral, vol. 9, pp. 82-88, 2004.

[15] S. Wiriosuparto, N. Krassilnik, S. Bhuta, J. Rao, and S. Firschowitz, "Solitary fibrous tumor: report of a case with an unusual presentation as a spindle cell parotid neoplasm," Acta Cytologica, vol. 49, no. 3, pp. 309-313, 2005.

[16] H. J. Kim, H. K. Lee, J. J. Seo et al., "MR imaging of solitary fibrous tumors in the head and neck," Korean Journal of Radiology, vol. 6, no. 3, pp. 136-142, 2005.

[17] S. S. Sreetharan and N. Prepageran, "Benign fibrous tumour of the parotid gland," Asian Journal of Surgery, vol. 28, no. 1, pp. 45-47, 2005.

[18] G. J. Ridder, G. Kayser, C. B. Teszler, and J. Pfeiffer, "Solitary fibrous tumors in the head and neck: new insights and implications for diagnosis and treatment," The Annals of Otology, Rhinology, and Laryngology, vol. 116, no. 4, pp. 265-270, 2007.

[19] A. Takahama Jr., J. E. Leon, O. P. de Almeida, and L. P. Kowalski, "Nonlymphoid mesenchymal tumors of the parotid gland," Oral Oncology, vol. 44, no. 10, pp. 970-974, 2008.

[20] K. J. Cho, J. Y. Ro, J. Choi, S. H. Choi, S. Y. Nam, and S. Y. Kim, "Mesenchymal neoplasms of the major salivary glands: clinicopathological features of 18 cases," European Archives of Oto-Rhino-Laryngology, vol. 265, no. S1, pp. 47-S56, 2008.

[21] N. Manglik, S. Patil, and M. F. Reed, "Solitary fibrous tumour of the parotid gland," Pathology, vol. 40, no. 1, pp. 89-91, 2008.

[22] X. J. Yang, J. W. Zheng, W. M. Ye et al., "Malignant solitary fibrous tumors of the head and neck: a clinicopathological study of nine consecutive patients," Oral Oncology, vol. 45, no. 8, pp. 678-682, 2009.

[23] O. A. Messa-Botero, A. E. Romero-Rojas, S. I. Chinchilla Olaya, J. A. az-Perez, and L. F. Tapias-Vargas, "Primary malignant solitary fibrous tumor/hemangiopericytoma of the parotid gland," Acta Otorrinolaringologica Espanola, vol. 62, no. 3, pp. 242-245, 2011.

[24] J. N. Iyengar, M. Atmaram, D. Neeli, and S. Prasad, "Solitary fibrous tumor presenting as a mass in the parotid gland," Indian Journal of Pathology \& Microbiology, vol. 54, no. 3, pp. 612-613, 2011.

[25] M. G. Cristofaro, E. Allegra, and M. Giudice, "Two new localizations of solitary fibrous tumor in the italian population: parotid gland and oral cavity-review of the literature," Journal of Oral and Maxillofacial Surgery, vol. 70, no. 10, pp. 2360-2367, 2012.

[26] A.-A. Sousa, G.-R. Souto, I.-A. Sousa, R.-A. Mesquita, R.S. Gomez, and B.-C. Jham, "Solitary fibrous tumor of the parotid gland: case report," Journal of Clinical and Experimental Dentistry, vol. 5, pp. e208-e211, 2013.

[27] E. Alonso-Rodríguez, T. González-Otero, A. Castro-Calvo, E. Ruiz-Bravo, and M. Burgueño, "Parotid gland solitary fibrous tumor with mandibular bone destruction and aggressive behavior," Journal of Clinical and Experimental Dentistry, vol. 6, pp. e299-e302, 2014.

[28] M. M. Kwok, M. Subramaniyan, and S. W. Chan, "Solitary fibrous tumour of the parotid gland: a case report and review of the literature," Case Reports in Otolaryngology, vol. 2015, Article ID 741685, 3 pages, 2015. 
[29] R. Yu and R. Rebello, "Solitary fibrous tumor of the parotid gland: a case report," Iranian Journal of Otorhinolaryngology, vol. 27, pp. 401-405.

[30] Y. Zhou, J. Zheng, Q. Zhu, W. Xia, and S. K. Bhagat, "Solitary fibrous tumor of the salivary gland: a case report," Oncology Letters, vol. 11, no. 1, pp. 901-903, 2016.

[31] L. A. Doyle, M. Vivero, C. D. M. Fletcher, F. Mertens, and J. L. Hornick, "Nuclear expression of STAT6 distinguishes solitary fibrous tumor from histologic mimics," Modern Pathology, vol. 27, no. 3, pp. 390-395, 2014.

[32] I. Ganly, S. G. Patel, H. E. Stambuk et al., "Solitary fibrous tumors of the head and neck: a clinicopathologic and radiologic review," Archives of Otolaryngology - Head \& Neck Surgery, vol. 132, no. 5, pp. 517-525, 2006.

[33] D. W. J. Lim, T. S. H. Tan, J. L. Tan, and K. Venkateswaran, "Solitary fibrous tumour of the parotid gland: a case report and a 15-year literature review," AME case reports, vol. 3, 2019.

[34] N. Romano, A. Ferrari, M. Moroni et al., "Solitary fibrous tumor of the deep parotid gland," Ear, Nose \& Throat Journal, vol. 99, 2020. 